\title{
PARALLEL COMPUTING OF FLUID-STRUCTURE COUPLED ANALYSIS USING SUPG/PSPG AND ENRICHED FREE MESH METHOD
}

\author{
Shinsuke NAGAOKA ${ }^{1}$, Yasushi NAKABAYASHI ${ }^{2}$, Genki YAGAWA ${ }^{1}$
}

${ }^{1}$ Center for Computational Mechanics Research (CCMR), Toyo University, 2-36-5, Hakusan, Bunkyo-ku, Tokyo, 112-0001, Japan.

${ }^{2}$ Faculty of Information Science and Arts, Toyo University, 2100, Kujirai, Kawagoe-shi, Saitama, 350-8585, Japan.

\begin{abstract}
The paper proposes a new analysis method for fluid-structure problems, which has nodal consistency at the fluid-structure interface and its calculation efficiency and accuracy are high. The incompressible viscous fluid analysis method using the P1-P1 element based on SUPG/PSPG developed by Tezduyar et al. is used for fluid analysis, while the high-accuracy analysis method based on EFMM developed by the authors is adopted for structure analysis. As the common feature of these methods, it is possible to analyze a fluid or a structure rather accurately by using the first order triangular of tetrahedral elements. In addition, variables are exchanged exactly at the common nodes on the fluid-structure boundary without deteriorating accuracy and calculation efficiency due to the interpolation of variables between nodes. The Present method is applied to a fluid-structure interaction problem by simulating the deformation of a red blood cell. At this time, to solve a bottleneck of EFMM that is needed a lot of time to make a stiffness matrix, we introduced a parallel processing to EFMM.
\end{abstract}

Keywords: Fluid-Structure coupled analysis, Enriched Free Mesh Method, SUPG/PSPG stabilized FEM, Parallel processing

\section{INTRODUCTION}

The analysis accuracy of the FEM is known to be improved by using higher-order elements with mid-side nodes. If engineers try to improve analysis accuracy without using high-order elements, they usually employ finer finite elements in the analysis domain, which results in the increase of calculation time and memory consumption[1-3].

It is well recognized that, for the FEM-based structural analysis, higher-order elements are generally used to improve analysis results. On the other hand, for fluid analysis, by employing the Streamline Upwind / Petrov-Galerkin (SUPG) [4] method and the Pressure - Stabilizing / Petrov-Galerkin (PSPG) [5-7] method, it is possible to achieve good analysis results without using higher-order elements. 
When conducting analysis considering fluid-structure interaction effects, it is desirable that node locations are consistent on the interface between fluid and structure domain as shown in Figure. 1. But, when the fluid analysis method using the SUPG/PSPG stabilized FEM and the structural analysis method using higher-order elements are used at the same time, the locations of nodes on the interface between two analysis domain becomes in consistent (see Figure. 2), because the second-order elements with mid-side nodes are used for the structural field and first-order elements without mid-side nodes for the fluid field, although these elements are both triangular and tetrahedral in the case of the 2D problems and the 3D problems, respectively.

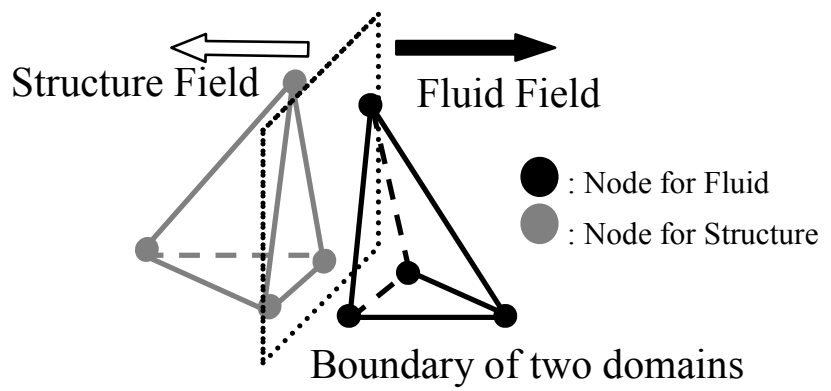

Figure 1. Boundary of two types of analysis field retaining elements formation consistent to each other

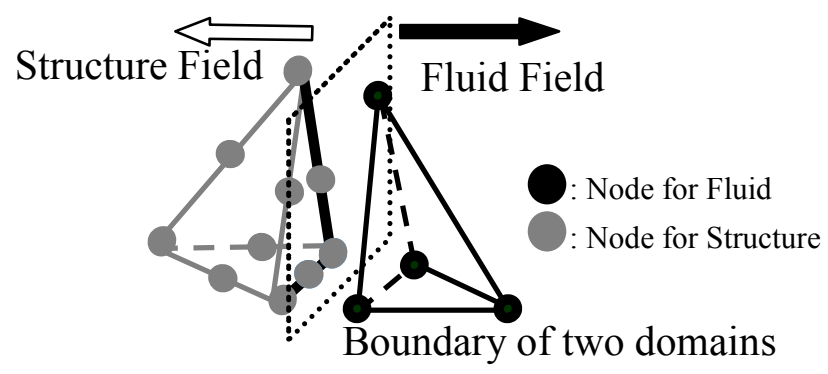

Figure 2. Boundary of two types of analysis field retaining elements formation inconsistent to each other incompatible boundary

Accordingly, when considering the coupling effects of two different fields, it is necessary to interpolate the analysis results between the nodes. Much has been accomplished in 3D computation of practical problem with incompatible meshes at the fluid structure interface (see, for example [8,9] ) and in some cases deliberate use of incompatible meshes might be part of the solution strategy (see, for example [10-13]). Still, using incompatible meshes at the interface complicates the calculation process, influences the coupling accuracy, and unless the solution strategy requires it, should be avoided. Therefore, when we try to perfume structurefluid coupled analysis, it is ideal to adopt an accurate structural analysis method without using the mid-side noded elements.

In order to cope with this problem, the authors propose to use the Enriched Free Mesh Method (EFMM) [14] as the structural analysis part, which is one of the meshless methods of high accuracy. The elements used for the EFMM based analysis are triangular or tetrahedral without mid-side nodes and it has been reported that the method gives solutions as accurate as that of the mid-side noded elements. By combining EFMM with the SUPG/PSPG stabilized FEM, it is possible to accurately analyze fluid-structure interaction problems in which the 
nodes on the boundaries between the structural and fluid fields are consistent as shown in Figure 1. The following section summarizes the EFMM with some numerical examples. To be more specific, verification of parallel efficiency and dynamic analysis are described. And the Sect. 3 describes the fluid analysis method with wimple numerical application to fluid structure interaction. Section 4 shows the application of the present method to a simulation of the deformation of blood cell. We conclude the present paper in Sect. 5.

\section{STRUCTURAL ANALYSI METHOD}

\subsection{Free Mesh Method}

EFMM used for the structural analysis in this study is based on FMM [15,16], which is one of mesh-less methods. The most important feature of FMM is that it requires only the coordinate data of each node in analysis domains as the input information. Based on the given coordinate data of nodes, a local triangular elements cluster is created at each node. To produce such a local triangular elements cluster, there are a variety of methods including the diagonal comparison method, the packaging method, and the method for developing a Delaunay triangle $[17,18]$ for each central node based on the planar relation between a Voronoi polygon and a Delaunay triangle.

Here, the node located at the center of a local triangular elements cluster is called the central node, while nodes located at the edge of the elements cluster are called the satellite nodes (see Figure. 3).

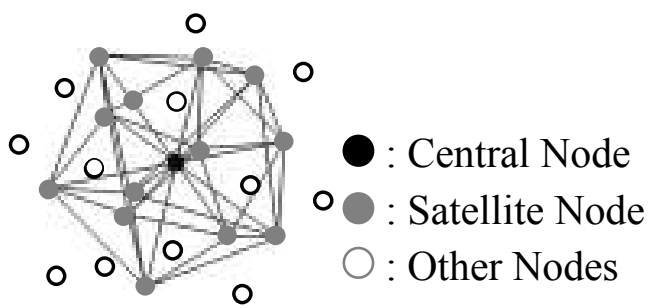

Figure 3. Fundamental concept of Free Mesh Method

The stiffness matrix of this local triangular elements cluster is calculated with the same way as the conventional FEM, and only the row components of the central node of the local triangular elements cluster are added to the global stiffness matrix. By performing this procedure for all the nodes in the analysis domain, it is possible to obtain the stiffness matrix for the global analysis domain. Then, the resulted final solution becomes equal to that of FEM.

\subsection{Enriched Free Mesh Method}

It is known that FMM has the difficulty in using mid-side noded elements due to its automatic element production algorithm. In order to overcome this problem with FMM, EFMM has been developed, where it is assumed that a local triangular elements cluster has an arbitrary strain field in addition to a displacement field like the conventional FEM or FMM (see Figure. 4). 


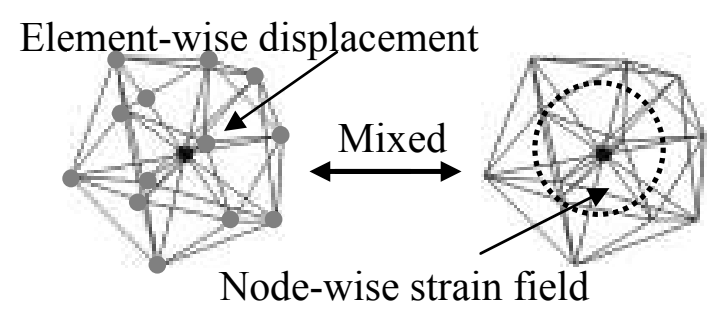

Figure 4. Two types of field postulated in local tetrahedral elements cluster

In EFMM, the displacement and the strain fields are, respectively, assumed in independent locations, and these two fields are linked with the Hellinger-Reissner principle[19]. The principle, in which displacement $u$ and strain $\varepsilon$ are assumed to be independent variables, is given by

$$
\Pi(\varepsilon, u)=\int_{\Omega}\{\varepsilon\}^{T}[D]\{\partial u\} d \Omega-\frac{1}{2} \int_{\Omega}\{\varepsilon\}^{T}[D]\{\varepsilon\} d \Omega-\int_{\Omega}\{u\}^{T}\{b\} d \Omega-\int_{S_{\sigma}}\{u\}^{T}\{\tilde{t}\} d S
$$

with

$$
\{\partial u\}=[B]\{\bar{u}\},\{u\}=\left[N^{u}\right]\{\bar{u}\},\{\varepsilon\}=\left[N^{\varepsilon}\right]\{\bar{\varepsilon}\}
$$

where $\{\bar{u}\}$ represents the nodal displacement, $\{\bar{\varepsilon}\}$ the unknown parameter of strain, $\{b\}$ the body force, $\{\tilde{t}\}$ the surface force on the boundary $\mathrm{S} \sigma$, and $\{\Omega\}$ the analysis domain. $[\mathrm{N} \varepsilon]$ is an arbitrary function that determines the strain of a local region, which can be is assumed as

$$
\left[N^{\varepsilon}\right]=\left[\begin{array}{cccccc}
p^{T} & 0 & 0 & 0 & 0 & 0 \\
0 & p^{T} & 0 & 0 & 0 & 0 \\
0 & 0 & p^{T} & 0 & 0 & 0 \\
0 & 0 & 0 & p^{T} & 0 & 0 \\
0 & 0 & 0 & 0 & p^{T} & 0 \\
0 & 0 & 0 & 0 & 0 & p^{T}
\end{array}\right]
$$

where

$$
p^{T}=\left[\begin{array}{llll}
1 & x & y & z
\end{array}\right]
$$

The stationary condition of Eq. (1) is expressed by

$$
\int_{\Omega} \delta\{\varepsilon\}^{T}[D]\left([B]\{\bar{u}\}-\left[N^{\varepsilon}\right]\{\bar{\varepsilon}\}\right) d \Omega=0
$$

and

$$
\int_{\Omega} \delta\{u\}^{T}[B]^{T}[D]\left[N^{\varepsilon}\right]\{\bar{\varepsilon}\} d \Omega-\int_{\Omega} \delta\{u\}^{T}\{b\} d \Omega-\int_{S_{\sigma}} \delta\{u\}^{T}\{\widetilde{t}\} d S=0
$$


These equations are written as follows,

$$
\left[\begin{array}{cc}
-A & C \\
C^{T} & 0
\end{array}\right]\left\{\begin{array}{l}
\bar{\varepsilon} \\
\bar{u}
\end{array}\right\}=\left\{\begin{array}{l}
f_{1} \\
f_{2}
\end{array}\right\}
$$

where

$$
\left\{\begin{array}{l}
A=\int_{\Omega}\left[N^{\varepsilon}\right]^{T}[D]\left[N^{\varepsilon}\right] d \Omega \\
C=\int_{\Omega}\left[N^{\varepsilon}\right]^{T}[D][B] d \Omega \\
f_{1}=0 \\
f_{2}=\int_{\Omega}\left[N^{u}\right]^{T}\{b\} d \Omega+\int_{\Gamma}\left[N^{u}\right]^{T}\{\tilde{t}\} d \Gamma
\end{array}\right.
$$

Next, Eq. (7) is condensed to the following equation:

$$
C^{T}\left(A^{-1} C \bar{u}\right)=f_{2}
$$

Finally, the local enriched stiffness matrix is derived as

$$
\left[k_{H R}\right]=C^{T} A^{-1} C
$$

Above enriched stiffness matrix is expected to give more accurate solutions than the usual FEM of FMM with the linear displacement base. The following section demonstrates that EFMM with the use of Eq. (10) is more accurate than the conventional FEM in a simple demonstrative example.

\subsection{Parallel Enriched Free Mesh Method}

EFMM is the technique that is based on FMM. FMM was proposed in order to obtain high parallel efficiency for analyzing by parallel FEM method. FMM and EFMM is nodebased analysis technique. It is already reported that parallel FMM analysis has a superiority of parallel efficiency compared with parallel FEM analysis. Though the process to make a stiffness matrix by parallel FMM is needed four times in the case of 3D analysis that is compared with conventional FEM.

Certainly EFMM and FMM is a node based analysis method, however, process to make a stiffness matrix by EFMM is different from FMM. The domain that is in the local elements cluster is used in the case of EFMM analysis. As a result, duplication of the calculation process of the stiffness matrix is eliminated. So, total computing time to make stiffness matrix by parallel EFMM of each node is as same as single EFMM. In other words, the improvement of the efficiency of parallelization will be higher depend on number of nodes.

In this section, result of verification of parallelization of EFMM that is using localelements-cluster by local-elements-cluster method is describing. As an example of verification of parallelization, cantilever model as shown in Figure 5 is used. Making time for stiff- 
ness matrix is compared with single processing and parallel processing for verification of the parallel efficiency.

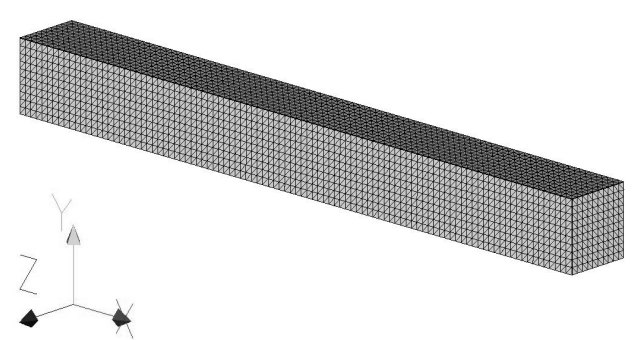

Number of nodes : 24000

Number of elements: 120000

Figure 5. Model for verification of parallel efficiency

Incidentally, Figure 6 is an example of model partitioning that is used for four parallel analysis. And Figure 7 shown the time to make stiffness matrix that is computed by single processing and parallel processing respectively. From this result, local-elements-cluster by local-elements-cluster method can be possible to obtain almost $100 \%$ parallelization efficiency. This numerical results mean that parallel efficiency will be higher as same as number of parallel units. This result means that this method become more effective by increasing number of parallel units.
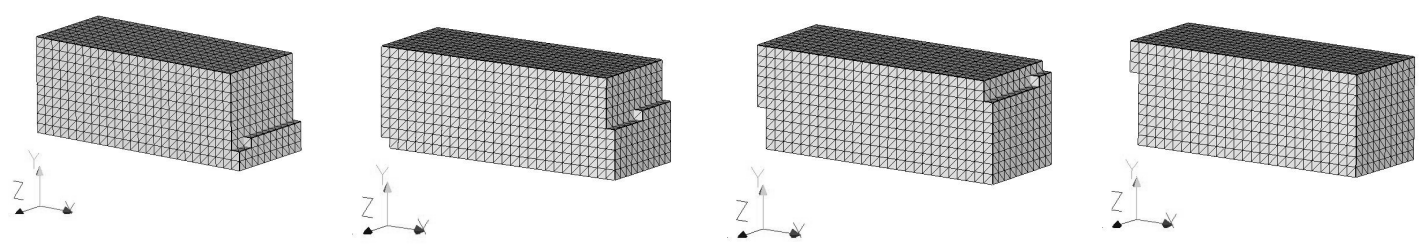

Figure 6. Example of domain decomposition

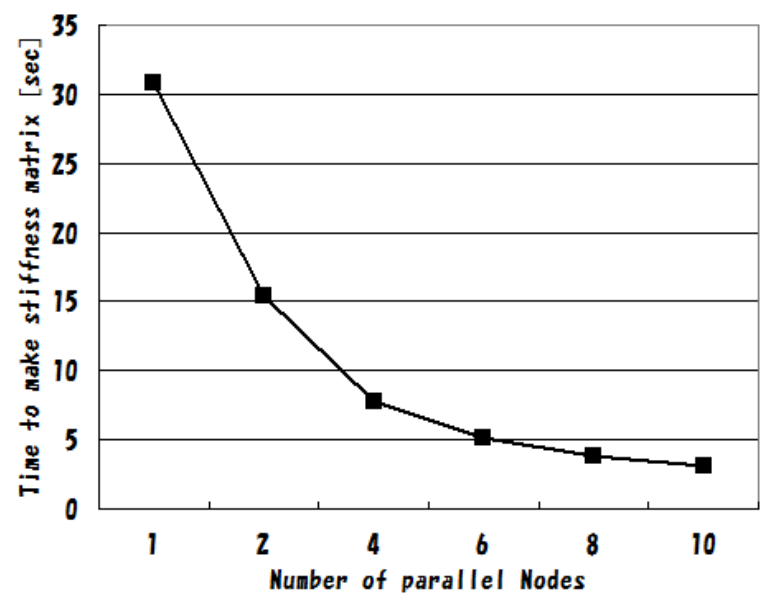

Figure 7. Relationship between number of parallel units and making time for stiffness matrix

A bottleneck of EFMM analysis is a process for making stiffness matrix. Because, this process is needed a lot of computation time compared with conventional FEM. Especially, in the case of dynamic problem by EFMM, stiffness matrix is needed a regeneration in every time step. As a result, this bottleneck become a very important issue and this problem can not be ignored considering the efficiency of the analysis. 
However, from this numerical result. High parallel efficiency as shown in above is obtained by parallelization using local-elements-cluster by local-elements-cluster treatment of EFMM is proof. From this result, conclusion shown in below is obtained. This treatment is very effective to solve a problem that is needed a regeneration of stiffness matrix in every time step like a dynamic problem.

\subsection{Numerical example for accuracy check}

In this section, the effectiveness of EFMM in dynamic analysis described using a numerical analysis case which has been compared with the accuracy of analysis with conventional FEM.

A stepwise vertical load is applied to a simply-supported beam as shown in Figure 8, and the time variation of displacement at the center of the beam is analyzed with EFMM and FMM, respectively.

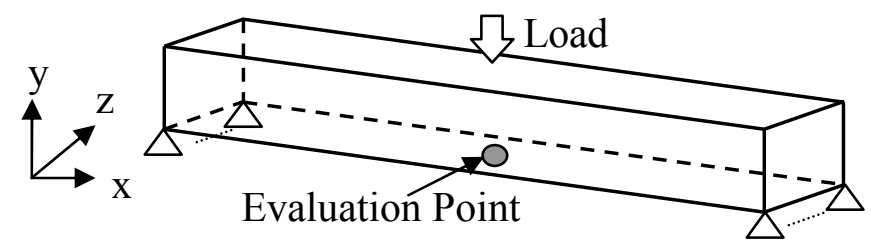

Figure 8. Analysis model

It is noted here that the solution by FMM is equivalent to that of the conventional FEM with linear displacement field. Mesh model used in this example is shown in Figure. 6. The theoretical solution of this example is given by the following equation,

$$
\begin{aligned}
& v(x, t)=\frac{2 P_{0} l}{\pi^{4} E I} \sum_{n=1}^{\infty} \frac{\alpha_{n}}{n^{4}}\left(1-\cos \omega_{n} t\right) \sin \frac{n \pi x}{l} \\
& \alpha_{n}=\left\{\begin{array}{cc}
1 & n=1,5,9 \ldots \\
-1 & n=3,7,11 \ldots \\
0 & n=2,4,6 \ldots
\end{array}\right.
\end{aligned}
$$

For time integration, Newmark's $\beta$ method [20] is adopted.

Figure 9 shows the example of analysis result computed by EFMM. Color counter in these figere mean 7-direction displacement.

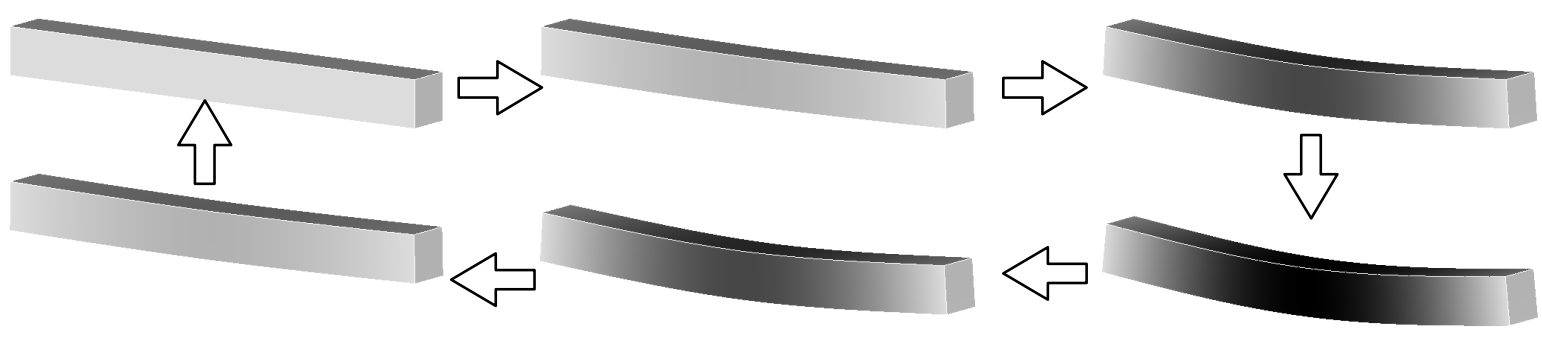

Figure 9. Example of analysis model 
Figure 10 shows the comparison between the result of FMM and EFMM-based analyes as well as the above theoretical solution, where the horizontal axis represents analysis time, and the vertical axis the displacement in the y-direction. This firure indicates that the result of the EFMM-based analysis is closer to the theoretical solution than that of the FMMbased analysis, although the same first-order tetrahedral element is used in these analyses.

From this result, it is confirmed that the use of EFMM can improve analysis accuracy without employing higher-order elements not only in static analysis but also in dynamic one.

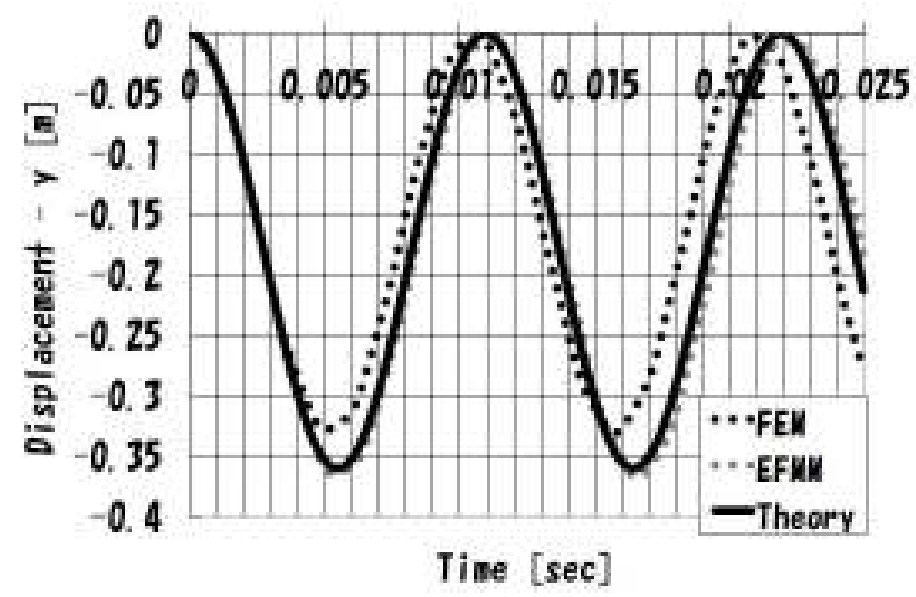

Figure 10. Analysis accuracy comparison between EFMM and FMM

\section{FLUID ANALYSIS METHOD}

\subsection{SUPG/PSPG method}

Here, the fluid analysis is conducted with a stabilized finite element formulation based on the SUPG[4] and PSPS[5-7] stabilizations (see [21] for a similar pressure stabilization method) that discretized the Navier-Stokes equation and the equation of continuity. As the basic equations for incompressible viscous fluid, the dimensionless Navier-Stokes equation and the incompressible continuity equation can be, respectively, written as follows,

$$
\begin{array}{ll}
\frac{\partial u_{i}}{\partial t}+u_{j} \frac{\partial u_{i}}{\partial x_{j}}+\frac{\partial p}{\partial x_{i}}-\frac{1}{\operatorname{Re}} \frac{\partial}{\partial x_{j}}\left(\frac{\partial u_{i}}{\partial x_{j}}+\frac{\partial u_{j}}{\partial x_{i}}\right)=0 & \text { in } \Omega \\
\frac{\partial u_{i}}{\partial x_{i}}=0 & \text { in } \Omega
\end{array}
$$

Where, $u_{i}$ and $p$ represent the velocity and the pressure, respectively. Re is the Reynolds number, and $\Omega$ is the analysis domain occupied by fluid. When the SUPG and PSPG method is applied to Eqs. (12) and (13), the following weak form is derived: 


$$
\begin{aligned}
\int_{\Omega} w_{i}\left(\frac{\partial u_{i}}{\partial t}+\bar{u}_{j} \frac{\partial u_{i}}{\partial x_{j}}\right) d \Omega-\int_{\Omega} \frac{\partial w_{i}}{\partial x_{i}} p d \Omega+\int_{\Omega} \frac{1}{\operatorname{Re}} \frac{\partial w_{i}}{\partial x_{j}}\left(\frac{\partial u_{i}}{\partial x_{j}}+\frac{\partial u_{j}}{\partial x_{i}}\right) d \Omega \\
+\sum_{e=1}^{n_{e l}} \int_{\Omega}\left(\tau_{s} \bar{u}_{k} \frac{\partial w_{i}}{\partial x_{k}}\right)\left(\frac{\partial u_{i}}{\partial t}+\bar{u}_{j} \frac{\partial u_{i}}{\partial x_{j}}+\frac{\partial p}{\partial x_{i}}\right) d \Omega=\int_{\Gamma_{h}} w_{i} h_{i} d \Gamma
\end{aligned}
$$

and

$$
\int_{\Omega} q \frac{\partial u_{i}}{\partial x_{i}} d \Omega+\sum_{e=1}^{n_{e l}} \int_{\Omega_{e}}\left(\tau_{p} \frac{\partial q}{\partial x_{i}}+\bar{u}_{j} \frac{\partial u_{i}}{\partial x_{j}}+\frac{\partial p}{\partial x_{i}}\right) d \Omega=0
$$

where $\mathrm{w}_{\mathrm{i}}$ and q represent the weighting functions of the Galerkin terms of Eqs. (12) and (13), respectively. $\Gamma$ denotes the boundary of the analysis domain $\Omega . \Gamma_{S}$ and $\Gamma_{p}$ represent the stabilization parameters of the SUPG and PSPG methods, respectively.

Regarding the velocity and the pressure, which are the unknown variables of Equ. (14) and (15), the interpolation is carried out with the first-order triangular elements. This combination is possible, because the formulation is based on the stabilization finite element method. Agter discretizing this formulation in the time direction, a system of linear equations is deribed with the unknown variable $\mathrm{U}^{\mathrm{n}+1}, \mathrm{~V}^{\mathrm{n}+1}$ and $\mathrm{P}^{\mathrm{n}+1}$ in the two-dimensional case. As the matrices in the above equations are asymmetric, we adopt the Generalized Product type method based on the Bi-CG)GPBi-CG) [22,23] method or the General Minimal Residual (GMRES(m)) method.

\subsection{Numerical example for accuracy check}

In this section, in order to prove the effectiveness of the SUPG/PSPG stabilized FEM that is using as a fluid analysis method of our coupled analysis method, we describe a numerical example using cavity model like a Figure 11.

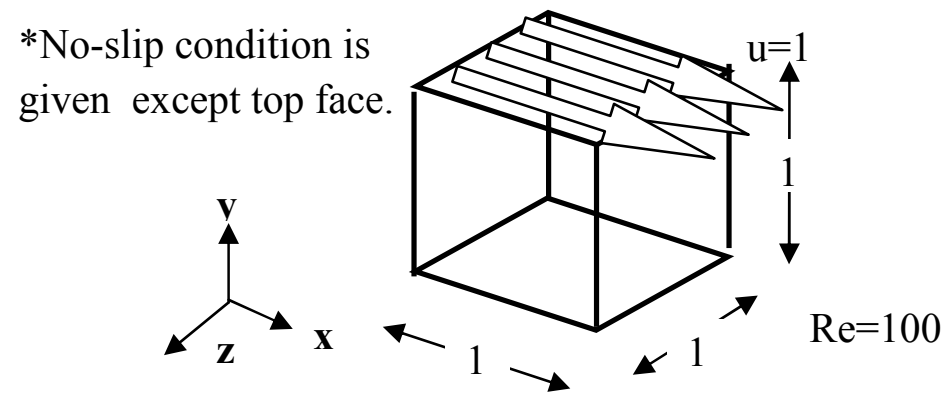

Figure 11. Analysis conditions

To prove this way, analysis result computed by SUPG/PSPG stabilized FEM is compared with experimental data by Ghia [24]. Analysis mesh model for this verification is as show in Figure 12. This model is created by about 9000 nodes and 48000 elements. 


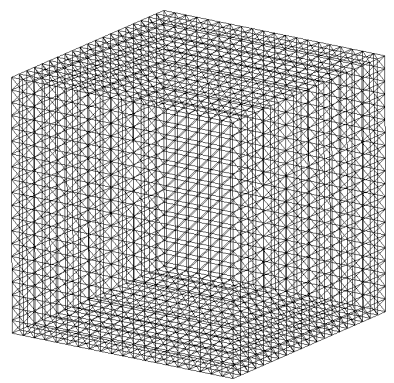

Number of Nodes : 9262

Number of Elements:48000

Figure 12. Analysis mesh model

Example of analysis result is introduced in Figure 13. From these results, it can be seen a behavior of fluid flow that is causing a convection in this cavity.
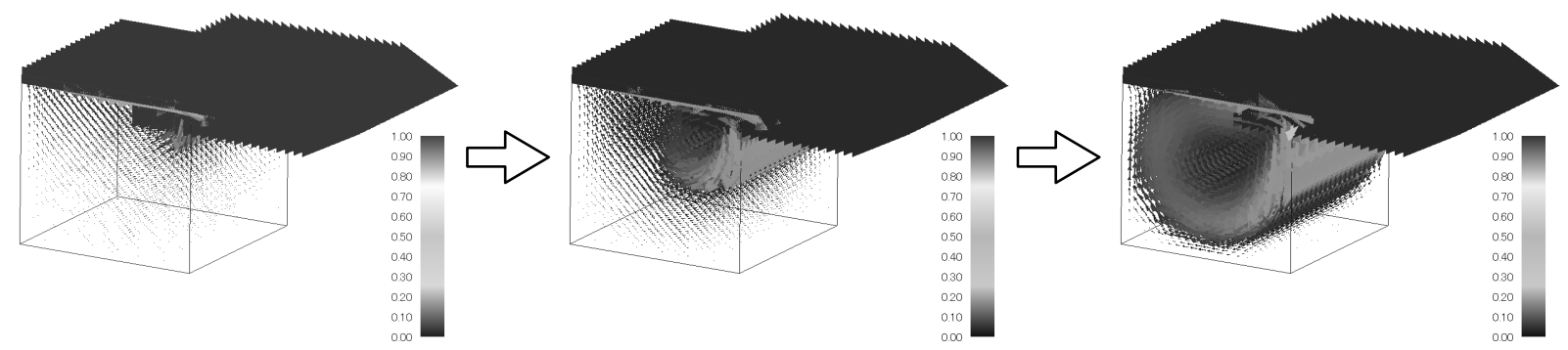

Figure 13. Example of analysis result

Analysis comparison between SUPG/PSPG stabilized FEM and experimental value by Ghia is as shown in Figure 14. Incidentally, lines in this graph mean x-direction velocity flow and $y$-direction velocity flow in the centerline of cavity. Analysis results compared with SUPG/PSPG stabilized FEM and experimental data by Ghia is nearly same.

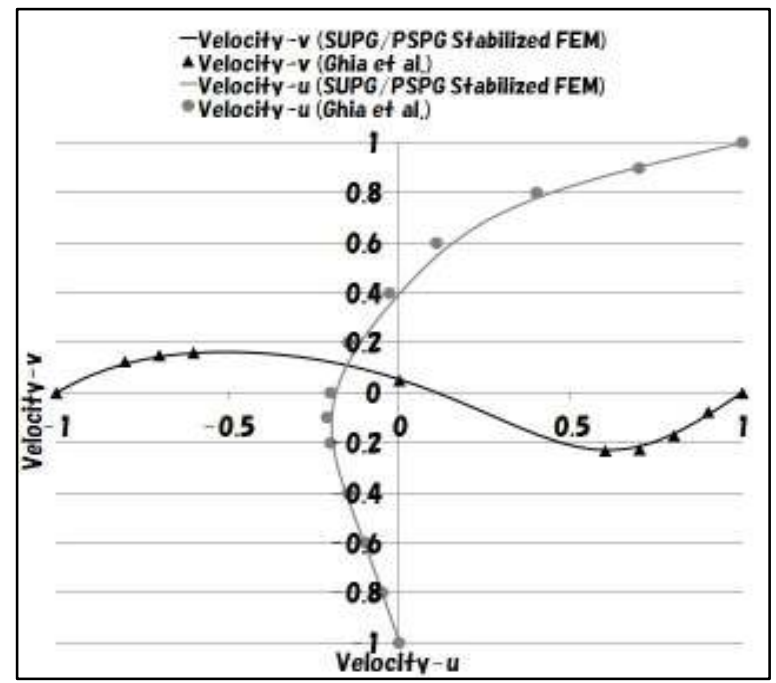

Figure 14. Example of analysis result

From these results, SUPG/PSPG stabilized FEM is proved as a suitable method for fluid analysis method of our new proposed coupled analysis method. 


\section{FLUID-STRUCTURE INTERACTION ANALYSIS}

In this study, we employ the so-called weak interaction analysis method, which is iterative and partitioned, considering that it is easy to use existing analysis codes and the convergence performance is rather high.

On the fluid-structure interface, the traction is given from the fluid side to the structure side, while the displacement or mesh moving velocity is given from the structure side to the fluid side. As mentioned above, in the proposed method, the nodes on the fluid-structure interface are totally consistent, which results that data exchange in the interface is conducted smoothly without any data interpolation between the nodes.

\subsection{Numerical example}

Here, we consider the analysis of the deformation of red blood cells in capillary vessels [25(37),26(38)] as a case study of the numerical analysis of a fluid-structure interaction, combining the structural analysis method based on EFMM and the fluid analysis method based on the SUPG/PSPG stabilized FEM using the ALE method [27-32(24-29)]. Here, ALE stands for Arbitrary Lagrangian-Eulerian.

For fluid-structure interaction analysis, it is generally necessary to integrate the Lagrangian structural analysis with the Eulerian fluid analysis. In the ALE method, the Eulerian and the Lagrangian descriptions are combined. By using the finite element mesh moving independently from the motion of fluid, it is possible to track the motion of fluid-structure interface without generating highly distorted elements. In addition, the governing equation for fluid expressed with the ALE method is the same as the Navier-Stokes equation for incompressible flow expressed with the Eulerian method, except that advection velocity is replaced with the relative velocity with respect to mesh moving speed. For these features the method, the ALE method can be considered to be appropriate for analyzing the fluid-structure interaction problems [33(30)].

A red blood cell has the characteristics to deform itself in order to pass through a blood vessel thinner than its size and moves through capillary vessels in the parachute shape by changing its shape. The original shape of a red blood cell is a disk with both sides being concave. This concave disk shape minimized the bending strain energy of the red blood cell membrane, and helps the easy deformation of the body. Figure 15 shows the details of the model used for the fluid analysis. It is assumed that the blood flows from the left hand side to the right hand side in the figure. The analysis is carried out with the no-slip condition at the capillary wall surface and the assumption that the blood is a Newtonian fluid. Figure 16 shows the details of the model used for the structure analysis. Incidentally, shape of a red blood cell is assumed ellipsoid and inside of red blood cell is assumed empty. 


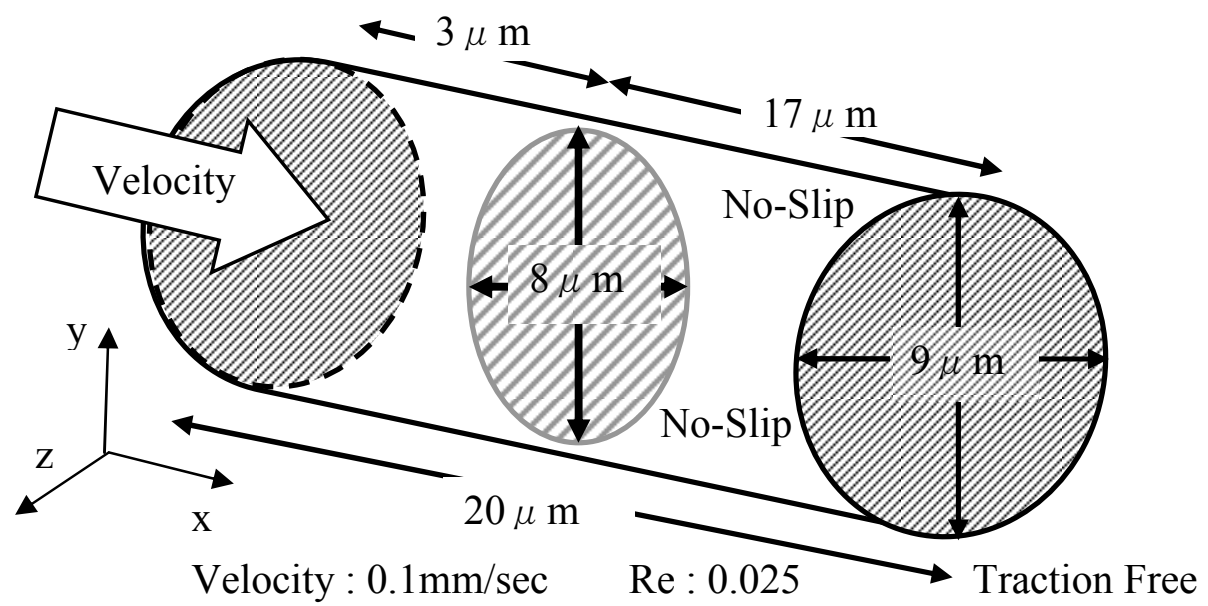

Figure 15. Analysis model and conditions of capillary (fluid analysis field)

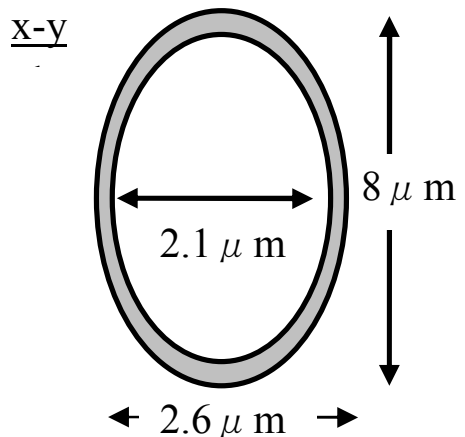

Young's modulus : $7500[\mathrm{~Pa}]$

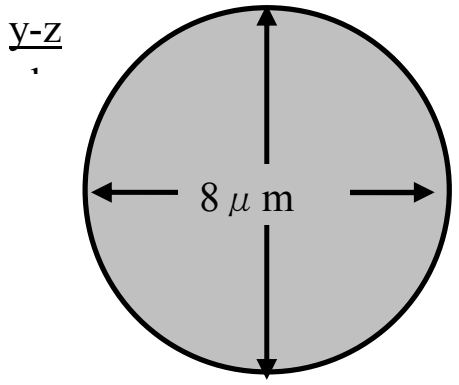

Mass : $33 p g$

Figure 16. Analysis model and conditions of a red blood cell (structure analysis field)

Figure 17 and Figure 18 show the finite element mesh for the fluid and structure fields at the initial state, respectively. The modes on the boundary between the fluid and the structure fields are allocated at the same positions.

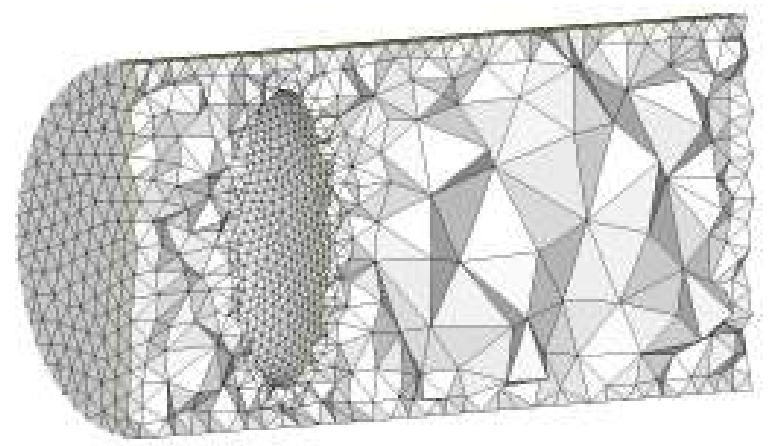

$\underline{x-y}$ plane

Number of Nodes : 11022 Number of Elements : 52929

Figure 17. Analysis mesh model of capillary (fluid analysis field) 


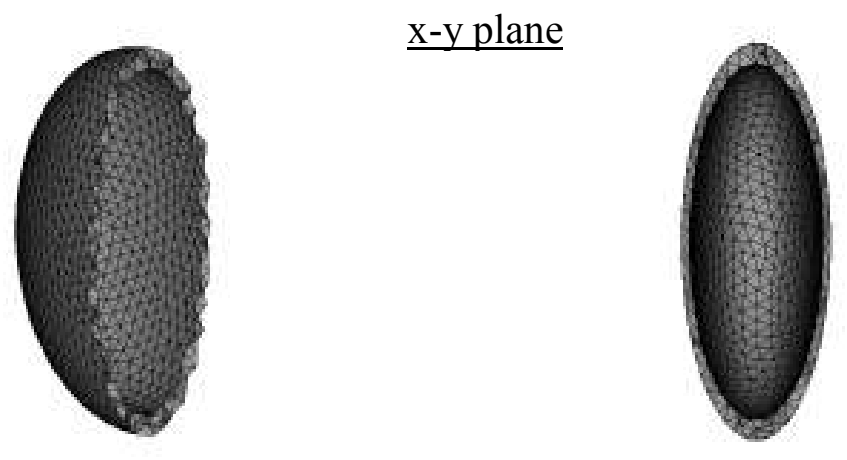

Number of Nodes : 4333 Number of Elements : 14570

Figure 18. Analysis mesh model of a red blood cell (structure analysis field)

Figure 19 shows the changing by time of the shape of red blood cell membrane and blood flow around the red blood cell membrane caused by fluid structure interaction. Color contour in these figure mean pressure.

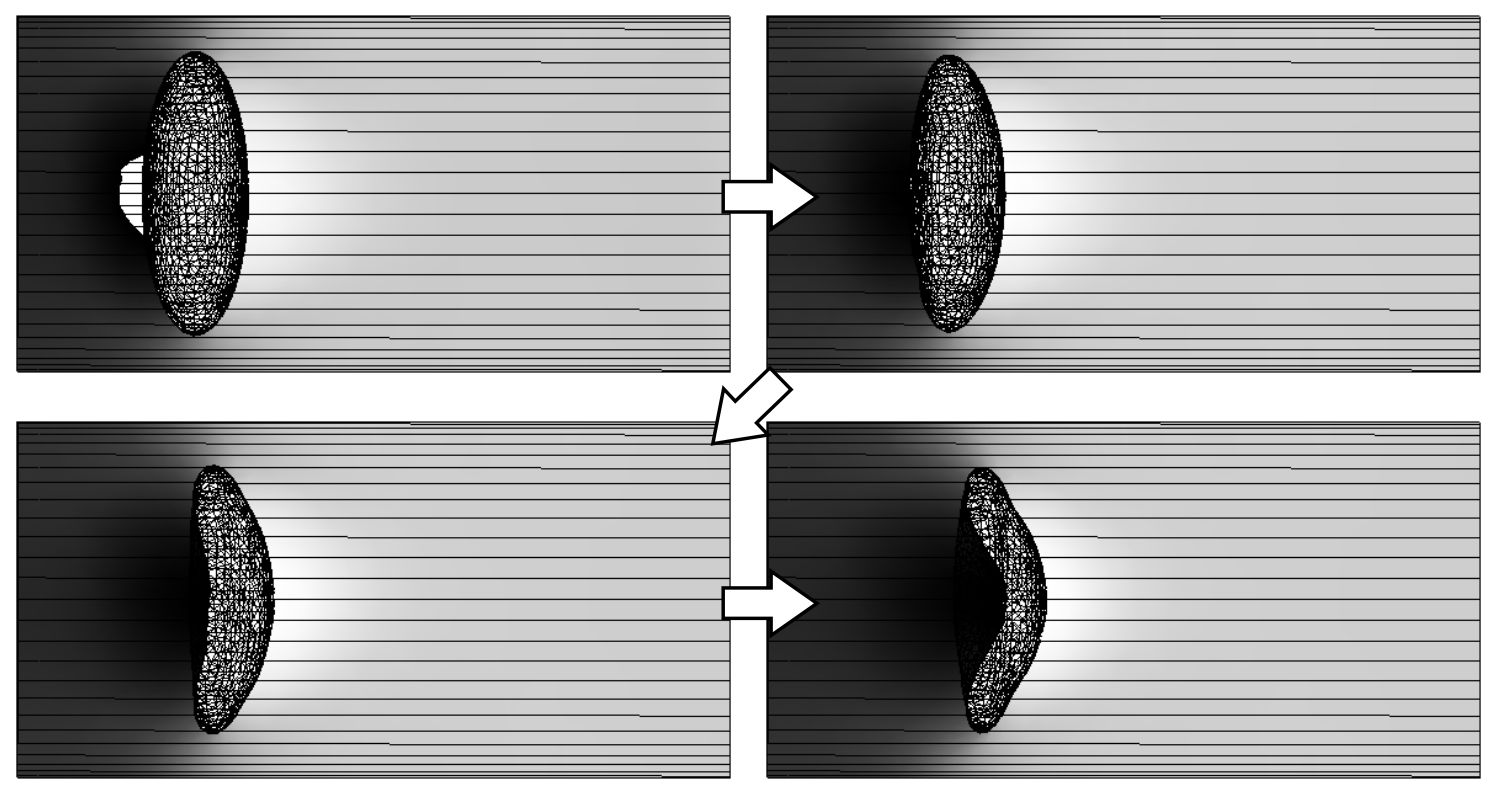

Figure 19. Changing of blood flow around red blood cell

Changing shape of the red blood cell membrane caused by pressure occurred by blood flow is described in Figure 20. The highest pressure is occurred central area of upstream of red blood cell membrane. Around the highest pressure area also appeared higher pressure. In other word, central area of upstream of red blood cell membrane is sunk by this pressure caused by blood flow. At the same time, blood flow in the capillary is changing significantly. As a result, shape of red blood cell membrane becomes like a parachute. 
Side
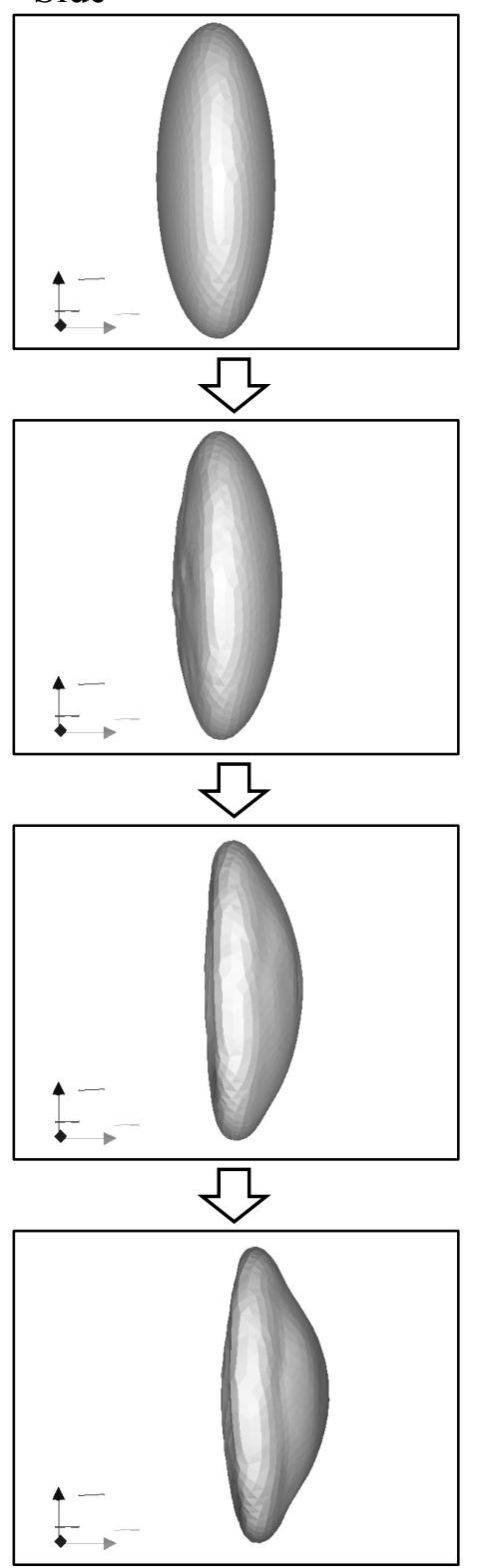

Front
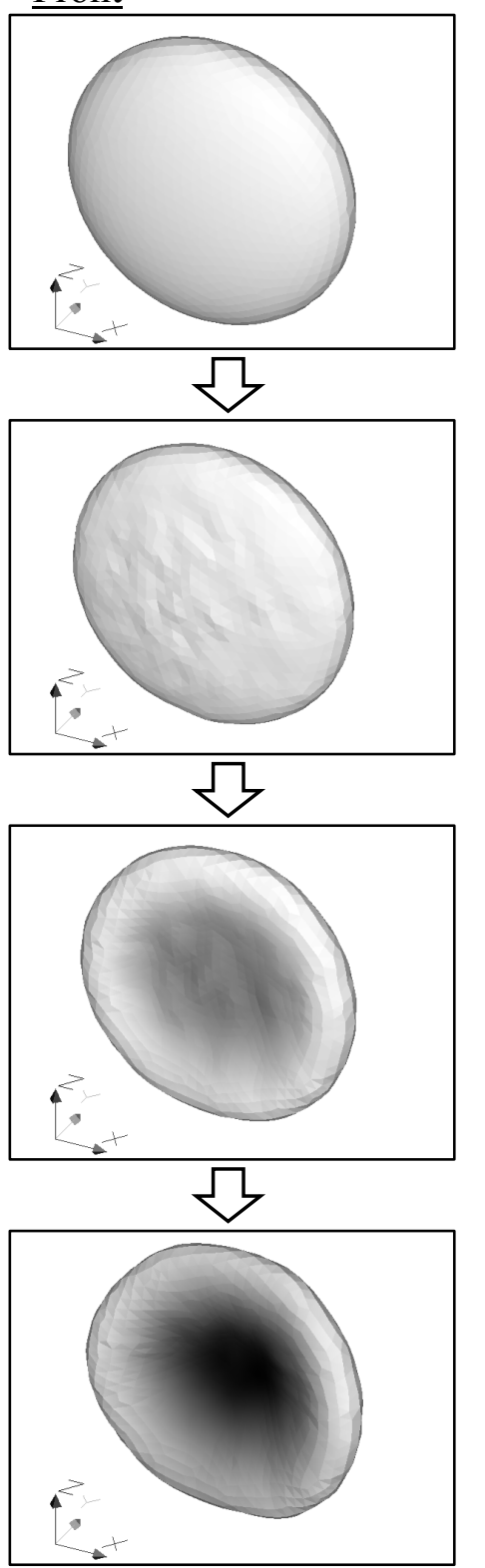

Back
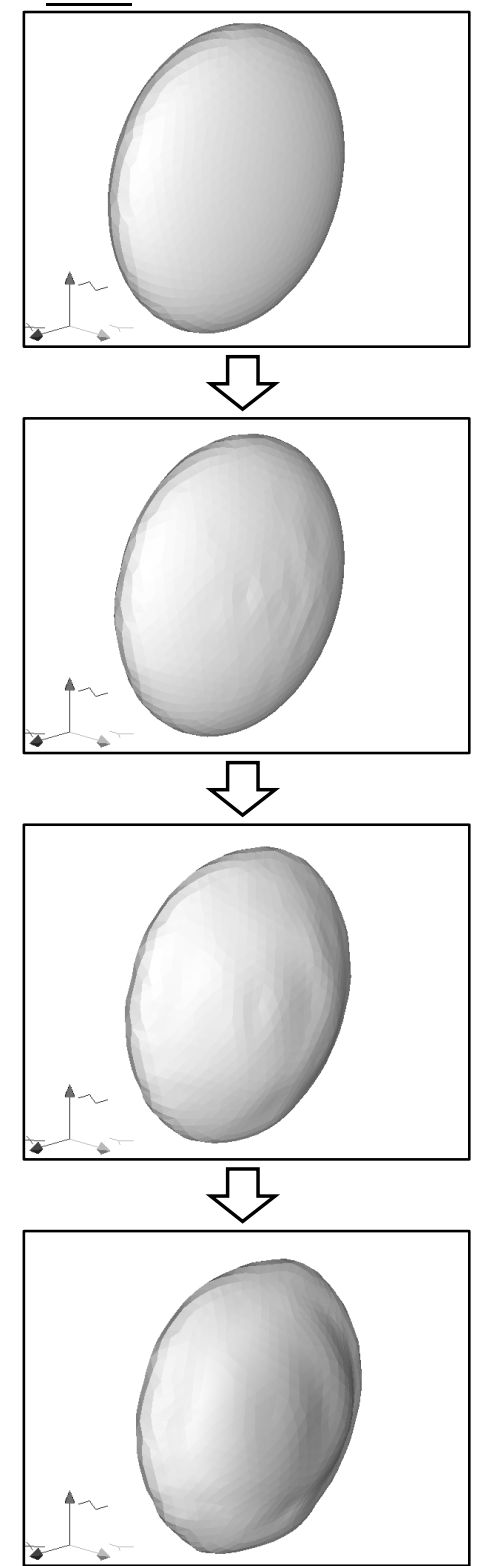

Figure 20. Deformation of a red blood cell membrane

Analysis result computed by our proposed method is as same as often observed in experiments and also in the human body. From these reasons, we prove that our proposed method can be possible to obtain fine analysis result, although quadratic elements are not used.

\section{CONCLUSION}

In this paper, the authors have proposed a fluid-structure analysis method without using the high-order elements by combining the EFMM-based structural analysis method and the SUPG/PSPG stabilized FEM-based fluid analysis method, in order to solve various problems with the numerical analysis considering the interaction effects.

As a realistic application of the present method. We show an analysis result of behavior of the red blood cell membrane in the capillary. Obtained analysis result is as same as behav- 
ior which is often observed in the human body. As a result, effectiveness of our proposed method is proved.

In the future work, we have to improve this method to compute a large scale problem. To be more specific, complete parallelization of the our proposed method is required. SUPG/PSPG stabilized FEM has been proven to be very high parallel efficiency is obtained. On the other hand, example of parallel EFMM is shown in above chapter. In this example, we introduced the parallel processing into the process that to make stiffness matrix. In the case of using EFMM, the process of creating a stiffness matrix becomes a bottleneck of the analysis process compared with conventional FEM. This problem was completely solved by parallelization of the process of creating a stiffness matrix computed by local-elements-cluster by local-elements-cluster, parallel efficiency to make the stiffness matrix that is shown in this paper was almost $100 \%$. From this result, high parallel efficiency will be obtained through the analysis process can be expected if EFMM become completely parallelization. As a result, our proposed coupled analysis method become high parallelization method and it will be possible to compute large scale problem easily.

\section{REFERENCES}

[1] Zienkiewicz OC, Taylor RL., "The finite element method". 5th edn. Elsevier, Amsterdam. 2000 .

[2] Hugher TJR. "The finite element method: linear static and dynamic finite element analysis". Prentice Hall, Newyork. 1987.

[3] Zienkiewicz OC, Taylor RL, Nifhiarasu P., "The finite element method for fluid dynamics". 6th edn. Elsevier, Amsterdam. 2005.

[4] Brooks AN, Hughes TJR., "Streamline upwind/Petrov Galerkin formulations for convection dominated flows with particular emphasis on the incompressible NavierStokes equations". Comput Method Appl Mech Eng 32:199-259. 1982.

[5] Tezduyar TE. "Stabilized finite element formulations for incompressible flow incompressible flow computations". Adv Appl Mech 28:1-44. 1992.

[6] Tezduyar TE, Mittal S, Ray SE, Shih R., "Incompressible flow computations with stabilized biliner and linear equal-order-interpolotion velocity-pressure elements". Comput Methods Appl Mech Eng 95:221-242. 1992.

[7] Tezduyar TE. "Computation of Moving Boundaries and Interfaces and Stabilization Parameters". Int J Numer Methods Fluids 43:555-575. 2003.

[8] Tezduyar TE, Sathe S, Keedy R, Stein K. "Space-time finite element techniques for computation of fluid-structure interactions". Comput Methods Appl Mech Eng 195:20022027.2006. 
[9] Texduyar TE, Sathe S., "Modeling of fluid-structure interactions with the space-time finite elements: solution techniques". Int J Numer Methods Fluids 54:855-900. 2007.

[10] Tezduyar TE, Sathe S, Pausewang J, Schwaab M, Christropher J, Crabtree J., "Interface projection techniques for fluid-structure interaction modeling with moving-mesh methods". Comput Mech 43:39-49. 2008

[11] Tezduyar TE, Takizawa K, Moorman C, Wright S, Christopher J., "Multiscale sequentially-coupled arterial FSI technique", Comput Mech 46:17-29. 2010.

[12] Takizawa K, Moorman C, Wright S, Christopher J, Tezduyar TE., "Wall shear stress calculations in space-time finite element computation of arterial fluid-structure interactions". Comput Mech 46:31-41. 2010.

[13] Takizawa K, Tezduyar TE., "Multiscale space-time fluid-structure interaction techniques". Comput Mech. doi:10.1007/s00466-011-0571-z. 2011.

[14] Yagawa G, Matsubara H., "Enriched free mesh method : an accuracy improvement for node-based fem, computational plasticity". Comput Methods Appl Sci 7:207-219. 2007.

[15] Yagawa G, Yamada T., "Free mesh method a new meshless finite element method", Comput Mech 18:383-386. 1996.

[16] Yagawa G, Yamada T., "Performance of Parallel computing of free mesh method". In:Proceedings of the 45th National Congress of Theroretical \& Applied Mechanics. 1996.

[17] Berg M, Cheong O, Kreveld M, Overmars M., "Computational geometry : algorithm and applicatioins. 3rd edn. Springer, New York. 2008.

[18] Inaga M, Fujisawa T, Okuda Y, Yagawa G., "Local mesh generation algorithm for free mesh method". The Japan Society of Mechanical Engineers [No.02-9] Dynamic and Desigh Conference . 2002.

[19] Zienkiewicz OC, Taylor RL., "Matrix finite element method I (Recision new publication)", Kagaku Gijutsu Shuppan, Inc. 1996.

[20] Newmark NM., "A method of computation for structural dynamics, civil engineering". Trans ASCE 127 : 1406-1435. 1962

[21] Franca LP, Frey SL., "Stabilized finite element methods II. The incompressible NavierStokes equations". Comput Methods Appl Mech Eng 99:209-233. 1992. 1406-1435. 1962

[22] Zhang S., "GPBi-CG generalized product-type methods based on Bi-CG for solving non-symmetriclinear system". SIAM J Sci Stat Comput 18:537-551. 1997 
[23] Thuthu M, Fujino S,. "Stability of GPBiCG AR method based on minimization of associate residual". ASCM 5081:108-120. 2008

[24] Ghia U, Ghia K.N, Shin C.T., "High-Re solutions for incompressible flow using the Navier-Stokes equations and a multigrid method. Journal of computational Physics, 48 , 387-411. 1982.

[25] Tanaka M, Sakamoto T, Sugawara S, Nagajima H, Katahira Y, Ohtsuki S, Kanai H., "Blood flow structure and dyanmics, and ejection mechanism in the left ventricle". $J$ Cardiol 52:86-101 . 2008.

[26] Kanno H, Morimoto M, Fujii H, Tsujimura T, Asai H, Noguchi T, Kitamura Y, Miwa S., "Primary structure of marine red blood cell-type Pyruvate Kinase (PK) and molecular characterization of PK deficiency identified in the CBA strain". Blood 86:3205-3210. 1995.

[27] Belytschko T, Flanagan DF, Kennedy JM., "Finite element method with user-controlled meshes for fluid-structure interactions". Comput Methods Appl Mech Eng 33:689-723. 1982.

[28] Huetra A, Liu WK., "Viscous flow with large free surface motion". Comput Methods Appl Mech Eng 69 : 277-324. 1988.

[29] Huetra A, Liu WK., "Viscous flow structure interaction". J press Vessel Technol 110:15-21. 1988.

[30] Nitikipaiboon C, Bathe KJ., "An arbitrary Lagrangian-Eulerian velocity potential formulation for fluid-structure interaction". Comput Struct 47:871-891. 1993.

[31] Bathe KJ, Zhang H, Wang MH., "A mixed displacement-based finite element formulation for acoustic fluid-structure interaction". Comput Struct $56: 225-237.1995$.

[32] Bathe KJ, Zhang H, Wang MH., "Finite element analysis of incompressible and compressible fluid flows with free interfaces and structural interactions". Comput Struct 56 : 193-213. 1995.

[33] Chakrabarti SK., "Fluid structure interaction and moving boundary problems". WIT Press, Southampton. 2007. 